\title{
Differential virial theorem in density-functional theory in terms of the Pauli potential for spherically symmetric electron densities: Illustrative example for the family of Be-like atomic ions
}

\author{
N. H. March ${ }^{1,2}$ and Á. Nagy ${ }^{3}$ \\ ${ }^{1}$ Department of Physics, University of Antwerp, Antwerp, Belgium \\ ${ }^{2}$ Oxford University, Oxford, England \\ ${ }^{3}$ Department of Theoretical Physics, University of Debrecen, H-4010 Debrecen, Hungary
}

(Received 12 August 2008; published 6 October 2008)

\begin{abstract}
The differential virial theorem relates the force $-\partial V / \partial \mathbf{r}$ associated with the one-body potential $V(\mathbf{r})$ of density-functional theory to the Laplacian $\nabla^{2} n$ of the ground-state density $n(\mathbf{r})$ and to a quantity $\mathbf{z}_{S}(\mathbf{r})$ involving the kinetic energy density tensor $t_{\alpha \beta}(\mathbf{r})$. Having the concept of the Pauli potential $V_{P}(\mathbf{r}), z_{s}$ is derived for spherically symmetric ground-state densities $n(r)$ in terms of the von Weizsäcker kinetic energy density and the first derivative of $V_{P}(r) . \mathbf{z}_{s}$ is related solely to the gradient kinetic energy density $t_{G}(r)$ for Be-like atomic ions.
\end{abstract}

DOI: 10.1103/PhysRevA.78.044501

PACS number(s): 31.15.E-

\section{BACKGROUND}

In earlier work with Gál [1], we obtained an explicit differential equation for the non-relativistic ground-state electron density $n(r, Z)$ for He-like atomic ions in the limit of large nuclear charge $Z e$, utilizing the work of Schwartz [2]. After a discussion of some implications of density-functional theory (DFT) [3] for spherically symmetric ground-state electron densities, our prime example will treat the Be-like ions cited in the title. We adopt here the approach via the differential virial theorem (DVT), going back to March and Young [4] for arbitrary level filling in one dimension and generalized first to spherically symmetric systems by Nagy and March [6] then to three dimensions by Holas and March [5]. Their result for the magnitude of $\partial V / \partial r$ of the force associated with the one-body potential $V(r)$ of DFT [3] reads, in spherical symmetry,

$$
-\frac{\partial V}{\partial r}=-\frac{\hbar^{2}}{4 m} \frac{(\partial / \partial r) \nabla^{2} n(r)}{n(r)}+\frac{z_{s}(r)}{n(r)} .
$$

The quantity $z_{s}(r)$ appearing in Eq. (1) is the singleparticle(s) limit of the many-electron vector field $\mathbf{z}(\mathbf{r})$ defined in [5] from the kinetic energy density tensor $t_{\alpha \beta}(\mathbf{r})$. In turn this latter quantity is defined from the first-order (manyelectron) density matrix $\gamma\left(\mathbf{r}^{\prime}, \mathbf{r}^{\prime \prime}\right)$ by

$$
t_{\alpha \beta}(\mathbf{r})=\frac{\hbar^{2}}{4 m}\left(\frac{\partial^{2}}{\partial r_{\alpha}^{\prime} \partial r_{\beta}^{\prime \prime}} \gamma\left(\mathbf{r}^{\prime}, \mathbf{r}^{\prime \prime}\right)+\frac{\partial^{2}}{\partial r_{\beta}^{\prime} \partial r_{\alpha}^{\prime \prime}} \gamma\left(\mathbf{r}^{\prime}, \mathbf{r}^{\prime \prime}\right)\right)_{\mathbf{r}^{\prime \prime}=\mathbf{r}^{\prime}=\mathbf{r}} .
$$

From $t_{\alpha \beta}(\mathbf{r})$ the explicit definition of the $\alpha$ component $z_{\alpha}(\mathbf{r})$ of the vector field $\mathbf{z}(\mathbf{r})$ introduced above is [5]

$$
z_{\alpha}(\mathbf{r})=2 \sum_{\beta} \frac{\partial t_{\alpha \beta}(\mathbf{r})}{\partial r_{\beta}}
$$

We note here that from the definitions (2) and (3) the quantity $z_{\alpha}(r)$ is, dimensionally, like a kinetic energy density divided by a length.

That this statement has a concrete consequence can be readily shown in the single-particle DFT limit of one occu- pied level. Then the single-particle Dirac density matrix $\gamma_{s}\left(\mathbf{r}, \mathbf{r}^{\prime}\right)$, appropriate to the He-like sequences of atomic ions, has the form in terms of the exact ground-state density $n$ :

$$
\gamma_{s}\left(r, r^{\prime}\right)=n(r)^{1 / 2} n\left(r^{\prime}\right)^{1 / 2} .
$$

Akbari et al. [7] have recently pointed out that if Eq. (4) is used in the definitions (2) and (3) above and applying the Euler equation (12) using the fact that the kinetic energy density is equal to the von Weizsäcker kinetic energy [Eq. (6) below], then $z_{s}(r)$ in Eq. (1) has the explicit form

$$
z_{s}(r)=4 \frac{t_{W}(r)}{r}+2 \frac{\partial t_{W}(r)}{\partial r},
$$

where $t_{W}(r)$ is the von Weizsäcker kinetic energy density [8] defined by

$$
t_{W}=\frac{\hbar^{2}}{8 m} \frac{\left(n^{\prime}\right)^{2}}{n} .
$$

Our major aim below is to generalize the result (5), which is exact for one-level occupancy only, to arbitrary level filling, provided always the resulting ground-state densities $n$ have spherical symmetry.

\section{GENERALIZATION OF EQ. (5) TO TAKE INTO ACCOUNT ARBITRARY LEVEL FILLING}

Before turning to the very specific example of Be-like atomic ions with configuration $(1 s)^{2}(2 s)^{2}$, we give below results concerning $z_{s}(r)$ in Eq. (1) for arbitrary level filling when the ground-state density $n$ is spherical.

The one-body potential $V(r)$ of DFT [3] leads to oneelectron wave functions $\psi_{i}(r)$ satisfying the Schrödinger equation

$$
\nabla^{2} \psi_{i}+\frac{2 m}{\hbar^{2}}\left[\varepsilon_{i}-V(r)\right] \psi_{i}=0
$$

Using the Laplacial form $t_{L}(r)$ of kinetic energy density for the appropriate general level occupancy, we multiply Eq. (7) by $\psi_{i}^{*}$ and sum over occupied levels to find 


$$
t_{L}(r)+n V(r)=\sum_{\text {occupied } i} \varepsilon_{i}\left|\psi_{i}\right|^{2} \equiv g(r) .
$$

Forming the gradient of Eq. (8) we find

$$
\nabla t_{L}(r)+n \nabla V(r)+V(r) \nabla n=\nabla g .
$$

Returning to Eq. (1) we can write

$$
z_{s}(r)=\frac{\hbar^{2}}{4 m} \frac{\partial}{\partial r} \nabla^{2} n(r)+\frac{\partial t_{L}}{\partial r}+V(r) \frac{\partial n(r)}{\partial r}-\frac{\partial g(r)}{\partial r} .
$$

Using Eq. (6) to replace $t_{L}(r)$ by the positive definite gradient form $t_{G}(r)$ of kinetic energy density, we readily obtain

$$
z_{s}(r)=\frac{\partial t_{G}(r)}{\partial r}+V(r) \frac{\partial n(r)}{\partial r}-\frac{\partial g(r)}{\partial r} .
$$

\section{INTRODUCTION OF PAULI POTENTIAL $V_{P}(R)$}

Several workers independenty introduced the concept of the Pauli potential $[9,10]$ termed $V_{P}(r)$ below. Using the Euler equation of DFT [3], namely

$$
\mu=\frac{\delta T_{s}}{\delta n(r)}+V(r)
$$

where $T_{s}[n]$ is the single-particle kinetic energy functional, we can write

$$
\frac{\delta T_{s}}{\delta n(r)}=\frac{\delta T_{W}}{\delta n(r)}+V_{P}(r) .
$$

Now the functional derivative of the von Weizsäcker kinetic energy $T_{W}$ is well known to have the form

$$
\frac{\delta T_{W}}{\delta n(r)}=\frac{\hbar^{2}}{8 m}\left(\frac{\nabla n}{n}\right)^{2}-\frac{\hbar^{2}}{4 m} \frac{\nabla^{2} n}{n} .
$$

Next let us replace $V(r)$ in Eq. (10) using Eq. (12) to find

$$
z_{s}(r)=\frac{\partial t_{G}}{\partial r}-\frac{\delta T_{s}}{\delta n(r)} \frac{\partial n(r)}{\partial r}+\mu \frac{\partial n(r)}{\partial r}-\frac{\partial g(r)}{\partial r} .
$$

Using Eqs. (13) and (14), Eq. (15) can be rewritten as

$$
\begin{aligned}
z_{s}(r)= & \frac{\partial t_{G}}{\partial r}-\frac{\hbar^{2}}{8 m}\left(\frac{n^{\prime}}{n}\right)^{2} n^{\prime}+\frac{\hbar^{2}}{4 m} \frac{\nabla^{2} n}{n} n^{\prime}-V_{P}(r) n^{\prime} \\
& +\mu \frac{\partial n(r)}{\partial r}-\frac{\partial g(r)}{\partial r} .
\end{aligned}
$$

If we write $t_{s}(r)$ as the sum of $t_{W}(r)$ and the Pauli contribution $t_{P}(r)$ then Eq. (16) becomes

$$
z_{s}(r)=2 \frac{\partial t_{W}}{\partial r}+4 \frac{t_{W}}{r}+\frac{\partial t_{P}}{\partial r}+\mu n^{\prime}-\frac{\partial g(r)}{\partial r}-V_{P}(r) n^{\prime} .
$$

In relation to Eq. (17) it may be useful to note a connection with the studies of Politzer and co-workers [11,12]. Using an "average" one-electron eigenvalue defined by $\bar{\varepsilon}(\mathbf{r})$ $=\sum_{i} \varepsilon_{i} n_{i}(\mathbf{r}) / n(\mathbf{r})$ and called local ionization potential, the quantity $g$ entering Eq. (17) can be replaced, when desired, by $g=\bar{\varepsilon} n$.
Now, we focus on replacing the quantity $\mu n^{\prime}-g^{\prime}$ entering the central Eq. (17) by Pauli quantities, and in particular by the derivative of the Pauli potential $V_{P}(r)$. From the oneelectron Schrödinger equation (7), one finds

$$
t_{L}(r)+n V(r)=g(r) .
$$

But from Eq. (12)

$$
n \frac{\delta T_{s}}{\delta n(r)}+n V(r)=n \mu .
$$

Furthermore, if we insert Eq. (13) into Eq. (19), we find

$$
n \frac{\delta T_{W}}{\delta n(r)}+n V_{P}(r)-t_{L}(r)=n \mu-g(r) .
$$

Using the explicit form of $\delta T_{W} / \delta n(r)$, given in Eq. (14), in Eq. (20) readily yields, after using Eq. (6),

$$
V_{P}(r)=\frac{t_{P}(r)}{n(r)}+\mu-\frac{g(r)}{n(r)} .
$$

Returning to Eq. (17), we can utilize Eq. (21) to remove $\mu n^{\prime}-g^{\prime}$ to obtain the desired result for $z_{s}(r)$ as

$$
z_{s}(r)=4 \frac{t_{W}}{r}+2 t_{W}^{\prime}(r)+n(r) V_{P}^{\prime}(r) .
$$

This reduces, as it must, to the one-level result (3) of Akbari et al. [7] when we set $V_{P}(r)=0$ for this case. [Note that Eq. (22) is an exact result, the approximation (4) was not applied in the derivation.] Equation (22) is a major focal point of this Brief Report.

\section{EXPLICIT EXAMPLE FOR TWO-LEVEL OCCUPANCY: THE CASE OF BE-LIKE ATOMIC IONS WITH NUCLEAR CHARGE ZE}

Here, since we are using throughout this Brief Report the single-particle limit of DFT [3] characterized by the onebody potential $V(r)$ in Eq. (1), $\mathbf{z}(\mathbf{r}) \rightarrow \mathbf{z}_{S}(\mathbf{r})$ for the Be-like series. In this limit, Dawson and March [13] wrote the Dirac first-order density matrix $\gamma_{s}\left(r, r^{\prime}\right)$ in a form generalizing the one-level result (4) to the Be-like series under discussion. Their form of $\gamma_{s}$ is constructed from a density amplitude $n(r)^{1 / 2}$ and a phase $\theta(r)$ as

$$
\gamma_{s}\left(r, r^{\prime}\right)=n(r)^{1 / 2} n\left(r^{\prime}\right)^{1 / 2} \cos \left[\theta\left(r^{\prime}\right)-\theta(r)\right] .
$$

Here the wave functions $\psi_{1 s}(r)$ and $\psi_{2 s}(r)$ are, respectively, $n(r)^{1 / 2} \cos \theta(r)$ and $n(r)^{1 / 2} \sin \theta(r)$. It is a straightforward, if somewhat tedious, matter to insert Eq. (23) into the definition (2) to obtain now the single-particle kinetic energy density tensor $t_{\alpha \beta}^{(s)}(\mathbf{r})$ in terms of density $n(r)$ and phase $\theta(r)$. Then the $\alpha$ component of $\mathbf{z}_{s}(\mathbf{r})$ introduced via Eq. (1) is also readily found by insertion of the above $t_{\alpha \beta}^{(s)}(\mathbf{r})$ in Eq. (3).

Rather than attempt to summarize the results of the above route, we outline below a simpler procedure based on Eq. (1). Thus we write 


$$
z_{s}(r)=\frac{\hbar^{2}}{4 m} \frac{\partial}{\partial r} \nabla^{2} n(r)-n(r) \frac{\partial V(r)}{\partial r} .
$$

Defining the Laplacian form $t_{L}(r)$ of the kinetic energy density from the wave functions $\psi_{1 s}(r)$ and $\psi_{2 s}(r)$ written above in terms of $n(r)$ and $\theta(r)$, it is a straightforward matter to use the Schrödinger equation (7) to find $\partial V / \partial r$ entering Eq. (24) as

$$
\frac{\partial V}{\partial r}=-\frac{t_{L}^{\prime}(r)}{n(r)}+\frac{t_{L}(r) n^{\prime}(r)}{n^{2}(r)}-\left(\varepsilon_{1 s}-\varepsilon_{2 s}\right) \theta^{\prime} \sin 2 \theta(r) .
$$

But it is well known [14] that $n(r)$ and $\theta(r)$ are related by a nonlinear pendulumlike equation, namely

$$
\nabla^{2} \theta(r)+\frac{\nabla n(r)}{n(r)} \nabla \theta(r)-2 \xi \sin 2 \theta(r)=0,
$$

where $\xi=\left(\varepsilon_{1 s}-\varepsilon_{2 s}\right) / 2$. Hence, using Eq. (26), the term involving $\sin 2 \theta(r)$ can be removed from Eq. (25) to obtain

$$
\begin{aligned}
\frac{\partial V}{\partial r}= & -\frac{t_{L}^{\prime}(r)}{n(r)}+\frac{t_{L}(r) n^{\prime}(r)}{n^{2}(r)} \\
& -\theta^{\prime}(r)\left[\theta^{\prime \prime}(r)+\frac{2}{r} \theta^{\prime}(r)+\frac{n^{\prime}(r)}{n(r)} \theta^{\prime}(r)\right] .
\end{aligned}
$$

We note that $t_{G}-t_{L}=\hbar^{2} / 4 m \nabla^{2} n(r)$ for use below. Next the phase terms in Eq. (27) can be removed by using the gradient form $t_{G}$ of the kinetic energy density. Inserting Eq. (27) in Eq. (24), using [13]

$$
t_{G}(r)=t_{W}(r)+\frac{1}{2} n\left(\theta^{\prime}\right)^{2},
$$

we can write the phase terms entering in Eq. (27) in terms of $\left[t_{G}-t_{W}\right] / n(r)$ and its first derivative. The result is a very straightforward generalization of the one-level form (5),

$$
z_{s}(r)=4 \frac{t_{G}(r)}{r}+2 \frac{\partial t_{G}(r)}{\partial r},
$$

which reduces immediately to Eq. (5) when we note that putting $\theta=0$ in the form of $\gamma_{s}$ in Eq. (23) leads back to the one-level form (4). It must not, of course, be assumed that Eq. (29) for the Be series of atomic ions will apply to higher level occupancy such as in the $\mathrm{Ne}$ atom with single-particle configuration $(1 s)^{2}(2 s)^{2}(2 p)^{6}$.

Briefly, one can, of course, regard Eq. (29) as a special case of the central Eq. (22). The Pauli potential $V_{P}(r)$ can be found from the work of Nagy [15] and is given by

$$
V_{P}(r)=\frac{1}{2}\left(\theta^{\prime}\right)^{2}-2 \xi \cos ^{2} \theta,
$$

where $\xi$ is defined immediately below Eq. (26). Some manipulation after forming $V_{P}^{\prime}(r)$ for insertion in Eq. (22) leads back to the intuitively appealing result (29), as the appropri- ate generalization of the one-level formula (5) for $z_{s}(r)$ entering the force balance Eq. (1).

\section{SUMMARY AND FUTURE DIRECTIONS}

The main results of the present study, with a starting point of the differential virial theorem [5] in DFT, are embodied, for spherically aymmetric electron densities, as follows:

(i) Equation (22) relating the magnitude of $\mathbf{z}_{s}(\mathbf{r})$ defined in Eq. (3) in terms of the single-particle first-order Dirac density matrix $\gamma_{s}\left(\mathbf{r}, \mathbf{r}^{\prime}\right)$, having by definition the exact ground-state density $n(r)$ as its diagonal, (i.e., $\mathbf{r}^{\prime}=\mathbf{r}$ ) to a sum of the three terms, involving the von Weizsäcker kinetic energy density $t_{W}(r)$ and the first derivative of the Pauli potential $V_{P}(r)$.

(ii) The fully worked out example of the family of Be-like atomic ions with nuclear charge $Z e$. If $t_{G}(r)$ is the gradient form of the single-particle kinetic energy density, $z_{s}(r)$ is shown to have the form $4 \frac{t_{G}(r)}{r}+2 \frac{\partial t_{G}(r)}{\partial r}$, which is an immediate generalization of the one-level result of Akbari et al. [7], where $t_{G}(r)$ becomes the von Weizsäcker form $t_{W}=\frac{\hbar^{2}}{8 m} \frac{\left(n^{\prime}\right)^{2}}{n}$.

As to future directions which should prove fruitful, the most immediate further application would be to utilize the shape of $\gamma_{s}\left(\mathbf{r}, \mathbf{r}^{\prime}\right)$ for the ground-state electron density $n(r, Z)$ for Ne-like atomic ions having nuclear charge $\mathrm{Ze}$. In the course of such an investigation, attention should clearly be given to the explicit construction of the Pauli potential $V_{P}(r)$. Finally, and in longer terms, Holas and March [5] compared and constucted the DVT for DFT with (as yet unknown!) potential $V(r)$ with the exact correlated result involving the density matrix $\gamma\left(\mathbf{r}, \mathbf{r}^{\prime}\right)$ satisfying $\gamma^{2}<\gamma$, in contrast to the idempotent Dirac matrix $\gamma_{s}$ used in the present paper. Returning briefly to [1], the differential equation for $n(r, Z)$ derived here should be used in the exact force-balance equation replacing the DFT form (1),

$$
-\frac{\partial V_{e x t}}{\partial \mathbf{r}}=-\frac{\hbar^{2}}{4 m} \frac{(\partial / \partial \mathbf{r}) \nabla^{2} n(\mathbf{r})}{n(\mathbf{r})}+\frac{\mathbf{z}(\mathbf{r})}{n(\mathbf{r})}+\mathbf{F}_{e e}(\mathbf{r}) .
$$

Hall, Jones, and Rees [16] have given a form of $\gamma\left(\mathbf{r}, \mathbf{r}^{\prime}\right)$ generalizing off the diagonal Schwartz electron density [2] utilized in [1]. This should throw light on the sum of the final two terms of Eq. (31), determined, respectively, from $\gamma\left(\mathbf{r}, \mathbf{r}^{\prime}\right)$, now used in the many-body definition of $t_{\alpha \beta}(\mathbf{r})$ in Eq. (2) by merely replacing $\gamma_{s}\left(\mathbf{r}, \mathbf{r}^{\prime}\right)$ by $\gamma\left(\mathbf{r}, \mathbf{r}^{\prime}\right)$ in [16]. A byproduct of such an investigation may throw further light on the correlation kinetic energy density $t_{c o r r}[n]$, to be added to the form $t_{G}(r)$ studied in this Brief Report.

\section{ACKNOWLEDGMENTS}

N.H.M. acknowledges that his contribution to this study was brought to fruition during a visit to the Department of Theoretical Physics, University of Debrecen. Á.N. acknowledges OTKA Grant No. T 67923. 
[1] T. Gál, N. H. March, and Á. Nagy, Chem. Phys. Lett. 305, 429 (1999).

[2] C. Schwartz, Ann. Phys. (N.Y.) 6, 156 (1959).

[3] R. G. Parr and W. Yang, Density Functional Theory of Atoms and Molecules (Oxford University Press, New York, 1989).

[4] N. H. March and W. H. Young, Nucl. Phys. 12, 237 (1959).

[5] A. Holas and N. H. March, Phys. Rev. A 51, 2040 (1995).

[6] Á. Nagy and N. H. March, Phys. Rev. A 40, 554 (1989).

[7] A. Akbari, N. H. March, and A. Rubio (unpublished).

[8] C. F. Weizsäcker, Z. Phys. 96, 341 (1935).

[9] N. H. March, Phys. Lett. 113A, 476 (1986).

[10] M. Levy, J. P. Perdew, and V. Sahni, Phys. Rev. A 30, 2745
(1984).

[11] P. Politzer, J. S. Murray, M. E. Grice, T. Brinck, and S. Ranganathan, J. Chem. Phys. 95, 6699 (1991).

[12] J. S. Murray, J. M. Seminario, P. Politzer, and P. Sjoberg, Int. J. Quantum Chem. 24, 645 (1990).

[13] K. A. Dawson and N. H. March, J. Chem. Phys. 81, 5850 (1984).

[14] See, for example, Á. Nagy and N. H. March, Phys. Rev. A 39, 5512 (1989).

[15] Á. Nagy, Chem. Phys. Lett. 460, 343 (2008).

[16] G. G. Hall, L. L. Jones, and D. Rees, Proc. R. Soc. London, Ser. A 283, 194 (1965). 